\title{
Percutaneous renal artery stent implantation in the treatment of atherosclerotic renal artery stenosis
}

\author{
YOUBIN HU $^{1 *}$, YONGGUANG ZHANG $^{1 *}$, HUA WANG $^{2}$, YONG YIN $^{2}$, \\ CHUNHUA CAO ${ }^{3}$, JING LUO ${ }^{4}$ and YUNFEI WANG ${ }^{5}$
}

\begin{abstract}
Departments of ${ }^{1}$ Cardiology, ${ }^{2}$ Obstetrics and Gynecology and ${ }^{3}$ Nephrology, Taizhou Hospital of Traditional Chinese Medicine, Taizhou, Jiangsu 225300; ${ }^{4}$ Department of Cardiology, Nanjing Zhongda Hospital, Nanjing, Jiangsu 210009;

${ }^{5}$ Department of Acupuncture, Taizhou Hospital of Traditional Chinese Medicine, Taizhou, Jiangsu 225300, P.R. China
\end{abstract}

Received January 12, 2018; Accepted June 26, 2018

DOI: $10.3892 / e t m .2018 .6440$

\begin{abstract}
We aimed to investigate the clinical curative effect of percutaneous renal artery stent implantation (PTRAS) in the treatment of atherosclerotic renal artery stenosis (ARAS), and to analyze the factors influencing the curative effect of PTRAS. A total of 230 patients with unilateral or bilateral renal artery stenosis were retrospectively analyzed. According to whether adverse cardiogenic or nephrogenic events occurred, 230 patients were divided into two groups to analyze the risk factors of adverse cardiogenic or nephrogenic events. The blood pressure of patients at each time-point after operation was decreased significantly compared with that before operation $(\mathrm{P}<0.01)$. The levels of serum creatinine $(\mathrm{SCr})$ at $24 \mathrm{~h}$ and 36 months after PTRAS were slightly increased compared with that before operation $(\mathrm{P}>0.05)$. The estimated glomerular filtration rate (eGFR) at each time-point after operation was slightly decreased compared with that before operation, but the difference was not statistically significant $(\mathrm{P}>0.05)$. Renography showed that GFR on the side of stent implantation at 36 months after PTRAS had no significant change compared with that before operation ( $\mathrm{P}>0.05$ ), but GFR on the unaffected side without receiving PTRAS was significantly increased compared with that before operation $(\mathrm{P}=0.0014)$. During the 36-month follow-up, there were a total of 56 cases of adverse cardiogenic or nephrogenic events. Multivariate regression analysis results showed that adverse cardiogenic or nephrogenic events after PTRAS were obviously associated with age ( $\geq 65$ years old), Charlson comorbidity index (CCI) score ( $\geq 2$ points), diabetes mellitus, stroke and congestive heart
\end{abstract}

Correspondence to: Dr Yunfei Wang, Department of Acupuncture, Taizhou Hospital of Traditional Chinese Medicine, 86 East Jichuan Road, Hailing, Taizhou, Jiangsu 225300, P.R. China

E-mail: wangyunfeicoffee@163.com

*Contributed equally

Key words: atherosclerotic renal artery stenosis, percutaneous renal artery stent implantation, hypertension failure $(\mathrm{CHF})(\mathrm{P}<0.05)$. In conclusion, PTRAS can effectively control the blood pressure and reduce the types of antihypertensive drugs used by patients with ARAS, but it has no definitely protective effect on renal function. Age ( $\geq 65$ years old), CCI score ( $\geq 2$ points), diabetes mellitus, stroke and CHF are risk factors leading to adverse cardiogenic or nephrogenic events after PTRAS.

\section{Introduction}

With the changes in dietary structure and population aging in China, atherosclerosis has become the most common cause of renal artery stenosis. It is estimated that atherosclerosis accounts for about $70 \%$ in all causes of renal artery stenosis in China, and 90\% in Europe and America $(1,2)$. Studies have shown that the advanced age, hypertension, hyperlipidemia, diabetes mellitus, renal insufficiency and peripheral vascular disease are high-risk factors of atherosclerotic renal artery stenosis (ARAS), and ARAS has a relatively high prevalence rate in cardiovascular diseases (3-8). ARAS causes a decrease in renal blood flow, which activates the renin-angiotensin system, leading to elevated blood pressure, whereas progressive lumen stenosis may lead to renal ischemia, causing progressive renal parenchymal damage, decreased renal function and ultimately leading to renal failure $(9,10)$.

Percutaneous renal artery stent implantation (PTRAS) can relieve the renal artery stenosis, restore the renal blood flow, block the activation of renin-angiotensin-aldosterone system (RAAS), treat the renal artery stenosis hypertension (RASH), and retard the progression of nephropathy and renal insufficiency. Numerous studies have shown that PTRAS can be used in the treatment of ARAS-induced hypertension, improving the renal function of patients with ARAS. Therefore, PTRAS has been used by many clinicians in clinical practice (11-13). However, in many clinical randomized controlled trials evaluating the curative effects of simple drug therapy and intervention combined with drug therapy on ARAS in recent years, there are no significant benefits for ARAS patients in the improvement of renal function, adverse cardiovascular events and long-term survival rate after revascularization, and patients also need to bear the relevant surgical risk, so whether PTRAS can benefit ARAS patients clinically is still controversial (14-17). 
In this study, the curative effect of PTRAS on 230 patients with ARAS and the factors influencing the prognosis of PTRAS were retrospectively analyzed, so as to provide a reference for selecting the therapeutic scheme of patients with renal artery stenosis.

\section{Patients and methods}

Research subjects. Patients receiving renal arteriography in the Cardiovascular Intervention Center of the Catheterization Room, Department of Cardiology of Taizhou Hospital of Traditional Chinese Medicine (Taizhou, China) from January 2010 to September 2014 were enrolled. They were diagnosed with ARAS via renal arteriography combined with clinical data according to the International Classification of Disease, Ninth Revision, Clinical Modification (ICD-9-CM), and received renal arterial stent implantation. Patients were followed up to detect the general conditions, blood pressure, types of antihypertensive drugs used, serum creatinine ( $\mathrm{SCr}$ ), renogram, and adverse cardiogenic and nephrogenic events; 230 patients with complete data were followed up for 36 months. This study was approved by the Ethics Committee of Taizhou Hospital of Traditional Chinese Medicine (Taizhou, China). Signed written informed consents were obtained from the patients and/or guardians.

Inclusion and exclusion criteria. Noninvasive and invasive examinations were initially performed to confirm that renal artery stenosis had hemodynamic significance. Renal artery stenosis diameter $\geq 70 \%$ usually indicates the hemodynamic significance, and the physiological significance of mild stenosis depends on the resistance of peripheral renal vessels or renal autoregulation system conditions. In order to accurately determine the degree of stenosis in patients with critical stenosis (renal artery stenosis, 50-70\%), the trans-lesion pressure gradient can be measured; the peak systolic pressure difference $>10 \%$ or the mean pressure difference $>5 \%$ indicates the hemodynamic significance, which can be used as an indication of interventional therapy.

Inclusion criteria $(18,19)$ : Renal artery stenosis patients with hemodynamic significance who had the following clinical manifestations and definite indications for percutaneous renal angioplasty may benefit from the intervention treatment: hypertension in patients aged $<30$ years old or late-onset hypertension in patients aged $>60$ years old; hypertension remains uncontrollable after combined application of at least three different types of antihypertensive drugs (one of which is diuretics); abnormal changes in blood pressure, including malignant, refractory or resistant hypertension (malignant hypertension refers to hypertension complicated by acute target organ damage, such as acute renal failure, acute decompensated heart failure, hypopsia, and progression into grades III and IV retinopathy); inability to tolerate antihypertensive drugs; recent azotemia or further deterioration of renal function after taking angiotensin-converting enzyme inhibitors (ACEI) or adrenergic receptor-binding (ARB) antihypertensive drugs; renal atrophy or different sizes in both kidneys (with a difference of $>1.5 \mathrm{~cm}$ ); patients with unexplained deterioration of renal function; cardiac disorder syndrome: recurrent transient pulmonary edema and unstable angina secondary to left ventricular dysfunction.
Exclusion criteria: elderly patients with weak constitution; patients whose disease was difficult to be controlled; patients who could not tolerate the interventional surgery; patients who were in the active stage of arterial inflammation; patients with the long diameter of renal atrophy $<7 \mathrm{~cm}$ or intrarenal arterial resistance index $>0.8$; patients with a serious allergic history of contrast agent or cholesterol embolism; patients receiving renal artery anatomy thus not suitable for interventional therapy or interventional therapy affected by subsequent treatments.

Preoperative preparation. After admission, patients were asked about their medical history and received physical examinations, blood routine examination, biochemistry, coagulation, echocardiography, renal color ultrasound and renal imaging before operation to clear the condition of disease. Patients and their families were informed of the purpose, methods and risks of operation, and signed the informed consent. The meridian approach was established, antihypertensive and anti-platelet drugs were used routinely, and water and electrolyte balance was maintained. At 1 day before operation and on the day of operation, patients took orally $300 \mathrm{mg}$ aspirin and $300 \mathrm{mg}$ clopidogrel hydrogen sulfate, and $1 \mathrm{ml} / \mathrm{min}$ normal saline was given for hydration at $24 \mathrm{~h}$ before operation.

Renal artery stent implantation method. The femoral or radial artery was punctured to send the catheter to the renal artery lesions for arteriography, and then the degree of lesion (lumen diameter and lesion length) was assessed. The guide wire was pushed through the lesion vessel, and the balloon was sent to the lesion along the guide wire and expanded. The stent was sent along the guide wire to the renal vascular stenosis, followed by positioning, dilation of the balloon and release of the stent, so that the stent was fully adherent to the vessel. Arteriography was performed again to observe the effect of stent implantation. If there was no residual stenosis, thrombosis, vascular tear, vascular dissection and other complications, the guide catheter could be withdrawn under the guidance of guide wire. After the hemostasis of arterial puncture site by compression, the operation was finished. Postoperative residual stenosis $<30 \%$ indicated the successful operation, and stent diameter $\geq 50 \%$ indicated the in-stent restenosis.

Postoperative management. Electrocardiogram and blood pressure continued to be monitored, and $1 \mathrm{ml} / \mathrm{min}$ normal saline was given for hydration. Vasopressor was withdrawn after operation for $24 \mathrm{~h}$, and the changes in the blood pressure of patients were observed. Patients took $75 \mathrm{mg} /$ day clopidogrel hydrogen sulfate orally for at least 3 months, and $100 \mathrm{mg} /$ day aspirin orally for at least 36 months. The blood lipid and blood pressure management was also provided every month.

Observation indexes. Patients enrolled were followed up for 36 months after operation, and the following indexes were collected. The peripheral arterial pressure in left upper limb of patients was measured under a quiet state before and after renal artery stent implantation 3 times, and the averages were taken. The changes in the types of antihypertensive drugs used by the patients were recorded. SCr and renogram results of patients were monitored and recorded before and after renal artery stent implantation. Estimated glomerular 
filtration rate (eGFR) was calculated: in males eGFR $\left(\mathrm{ml} / \mathrm{min} / 1.73 \mathrm{~m}^{2}\right)=[175 \times \mathrm{SCr}(\mathrm{mg} / \mathrm{dl})-1.234 \times$ age $($ years old) - 0.179]; in females eGFR $\left(\mathrm{ml} / \mathrm{min} / 1.73 \mathrm{~m}^{2}\right)=[175 \mathrm{x} \mathrm{SCr}$ $(\mathrm{mg} / \mathrm{dl})-1.234 \mathrm{x}$ age (years old) - 0.179] x 0.79. Hypertension, diabetes mellitus, hyperlipemia, stroke and other complications in patients were recorded, and Charlson comorbidity index (CCI) (20) was scored for patients, and the comorbidities considered included diabetes mellitus, hyperlipemia, stroke, smoking history, and congestive heart failure (CHF). Whether adverse cardiogenic and nephrogenic events occurred within 36 months after renal artery stent implantation was detected (17), including death, myocardial infarction, stroke, hospitalization due to $\mathrm{CHF}$, deterioration of renal function (excluding other reasons, decrease of eGFR by $\geq 30 \%$ over the initial value for $\geq 60$ days), and permanent replacement therapy of renal function required.

Analyses of risk factors of adverse cardiogenic and nephrogenic events. Patients were divided into adverse event group and control group according to whether adverse cardiogenic and nephrogenic events occurred in patients. Multivariate regression analyses were performed with relevant factors of adverse events (including age, sex, smoking history, stroke, diabetes mellitus, hyperlipemia, congestive heart failure and CCI score) as variables.

Statistical analysis. Statistical Product and Service Solutions (SPSS) 19.0 software (IBM Corp., Armonk, NY, USA) was used for the analysis. Chi-square test was used for the comparison of OR values and t-test for the comparison of $\mathrm{SCr}$ and eGFR. Data are presented as mean \pm standard deviation to evaluate the curative effect of PTRAS. Logistic regression analyses were used to analyze the risk factors related to adverse cardiogenic and nephrogenic events after PTRAS. P $<0.05$ suggested that the difference was statistically significant.

\section{Results}

Baseline data of patients. Among a total of 230 cases enrolled, there were 128 males and 102 females aged 63.5 \pm 14.1 years old with a course of hypertension of $15.3 \pm 11.8$ years. Preprocedure, $2.8 \pm 1.2$ types of antihypertensive drugs were used, and there were 123 cases with a smoking history, 143 cases of hyperlipemia, 183 cases of coronary heart disease, 78 cases of diabetes mellitus, and 36 cases of stroke. A total of 286 stenotic renal arteries were treated, among which 56 cases received bilateral renal artery stent implantation, and 174 cases received unilateral renal artery stent implantation. The success rate of renal artery stent implantation was $100 \%$, and no serious complications related to operation occurred (Table I).

Changes in blood pressure before and after PTRAS. Before operation, the mean systolic blood pressure of patients was $158.7 \pm 20.5 \mathrm{mmHg}$, and the mean diastolic blood pressure was $92.6 \pm 10.1 \mathrm{mmHg}$. At $24 \mathrm{~h}$ after operation, the mean systolic blood pressure of patients was decreased to $131.4 \pm 13.8 \mathrm{mmHg}$, and the mean diastolic blood pressure was decreased to $71.7 \pm 8.5 \mathrm{mmHg}$. At 36 months after operation, the mean systolic blood pressure was $135.2 \pm 10.4 \mathrm{mmHg}$, and the mean diastolic blood pressure was $72.7 \pm 7.9 \mathrm{mmHg}(\mathrm{P}<0.01)$. The
Table I. Baseline characteristics of ARAS patients.

\begin{tabular}{lc}
\hline Parameters & Patients \\
\hline Sex & \\
$\quad$ Male, n (\%) & $128(55.7)$ \\
Female, n (\%) & $102(44.3)$ \\
Age (years) & $63.5 \pm 14.1$ \\
Systolic pressure (mmHg) & $158.7 \pm 20.5$ \\
Diastolic pressure (mmHg) & $92.6 \pm 10.1$ \\
Number of antihypertensive medications & $2.8 \pm 1.2$ \\
SCr ( $\mu$ mol/l) & $130.3 \pm 51.4$ \\
Smoking history, n (\%) & $123(53.5)$ \\
Hyperlipidemia, $\mathrm{n}(\%)$ & $143(62.2)$ \\
Coronary heart disease, $\mathrm{n}(\%)$ & $183(79.6)$ \\
Diabetes mellitus, $\mathrm{n}(\%)$ & $78(33.9)$ \\
Stroke, n (\%) & $36(15.7)$
\end{tabular}

ARAS, atherosclerotic renal artery stenosis; $\mathrm{SCr}$, serum creatinine.

blood pressure of patients at each time-point after operation was significantly decreased compared with that before operation $(\mathrm{P}<0.05)$. The number of types of antihypertensive drugs used was decreased from $2.8 \pm 1.2$ before operation to $2.0 \pm 0.9$ 36 months after operation $(\mathrm{P}<0.01)$ (Table II).

Changes in renal functions before and after PTRAS. SCr was increased in 123 cases $(53.5 \%)$ and decreased in 107 cases (46.5\%). The levels of SCr at $24 \mathrm{~h}(140.5 \pm 60.1 \mu \mathrm{mol} / \mathrm{l})$ and at 36 months $(133.2 \pm 55.6 \mu \mathrm{mol} / \mathrm{l})$ after PTRAS were slightly increased compared with that before operation $(130.3 \pm 51.4 \mu \mathrm{mol} / \mathrm{l})$, and there were no statistically significant differences $(\mathrm{P}>0.05)$. eGFR at each time-point after operation was slightly decreased compared with that before operation, but the difference was not statistically significant ( $\mathrm{P}>0.05$ ) (Table III). A total of 90 patients underwent renography at 1 year before and after operation to evaluate the renal functions. A total of 90 cases of renal artery stenosis $(>70 \%)$ were treated with stent implantation, including 54 cases of unilateral renal artery stenosis and 36 cases of bilateral renal artery stenosis. GFR on the side of receiving PTRAS had no significant change compared with that before operation (P>0.05), but GFR on the unaffected side without receiving PTRAS was significantly increased compared with that before operation ( $\mathrm{P}=0.0014)$ (Fig. 1).

Analyses of risk factors of adverse cardiogenic or nephrogenic events after PTRAS. During the follow-up period, there were a total of 56 cases of adverse cardiogenic or nephrogenic events $(24.3 \%)$, including 5 cases of all-cause death, 10 cases of myocardial infarction, 5 cases of stroke, 10 cases of hospitalization due to heart failure, 23 cases of deterioration of renal function, and 3 cases of permanent dialysis treatment (Table IV). Patients were divided into adverse event group and control group according to whether adverse cardiogenic and nephrogenic events occurred during the follow-up. 
Table II. Comparison of blood pressure before and after PTRAS.

\begin{tabular}{lccc}
\hline Time & Systolic pressure $(\mathrm{mmHg})$ & Diastolic pressure $(\mathrm{mmHg})$ & No. of antihypertensive medications \\
\hline Preprocedure & $158.7 \pm 20.5$ & $92.6 \pm 10.1$ & $2.8 \pm 1.2$ \\
Postprocedure & & & $2.1 \pm 1.1$ \\
$24 \mathrm{~h}$ & $131.4 \pm 13.8$ & $71.7 \pm 8.5$ & $2.2 \pm 1.0$ \\
12 months & $132.3 \pm 14.4$ & $72.9 \pm 8.7$ & $2.1 \pm 1.2$ \\
24 months & $133.9 \pm 12.6$ & $74.5 \pm 8.8$ & $2.0 \pm 0.9$ \\
36 months & $135.2 \pm 10.4$ & $72.7 \pm 7.9$ & \\
\hline
\end{tabular}

PTRAS, percutaneous renal artery stent implantation.

Table III. Comparison of renal function before and after PTRAS.

\begin{tabular}{lcc}
\hline Time & $\mathrm{SCr}(\mu \mathrm{mol} / \mathrm{l})$ & $\mathrm{eGFR}\left(\mathrm{ml} / \mathrm{min} / 1.73 \mathrm{~m}^{2}\right)$ \\
\hline $\begin{array}{l}\text { Preprocedure } \\
\text { Postprocedure }\end{array}$ & $130.3 \pm 51.4$ & $70.1 \pm 17.7$ \\
$24 \mathrm{~h}$ & $140.5 \pm 60.1$ & $67.3 \pm 15.2$ \\
12 months & $139.8 \pm 55.7$ & $68.4 \pm 17.5$ \\
24 months & $136.1 \pm 52.4$ & $69.3 \pm 18.9$ \\
36 months & $133.2 \pm 55.6$ & $68.8 \pm 19.1$
\end{tabular}

PTRAS, percutaneous renal artery stent implantation; SCr, serum creatinine; eGFR, estimated glomerular filtration rate.

Table IV. The occurrence of cardiovascular or renal adverse events after PTRAS.

Cardiovascular or renal adverse events

\begin{tabular}{lc}
\hline All-cause deaths & $5(8.9)$ \\
Myocardial infarction & $10(17.9)$ \\
Stroke & $5(8.9)$ \\
Heart failure & $10(17.9)$ \\
Deterioration of renal function & $23(41.1)$ \\
Permanent dialysis treatment & $3(5.4)$
\end{tabular}

PTRAS, percutaneous renal artery stent implantation.

Multivariate regression analyses of adverse cardiogenic or nephrogenic events after PTRAS showed that the occurrence of adverse events was obviously associated with advanced age ( $\geq 65$ years old), higher CCI score ( $\geq 2$ points), diabetes mellitus, stroke and CHF $(\mathrm{P}<0.05)$ (Table V).

\section{Discussion}

With the development of interventional therapy, renal artery stent implantation has become one of the main methods for the treatment of ARAS. PTRAS can restore the renal blood supply, block the damage of ischemia to the kidney and improve the

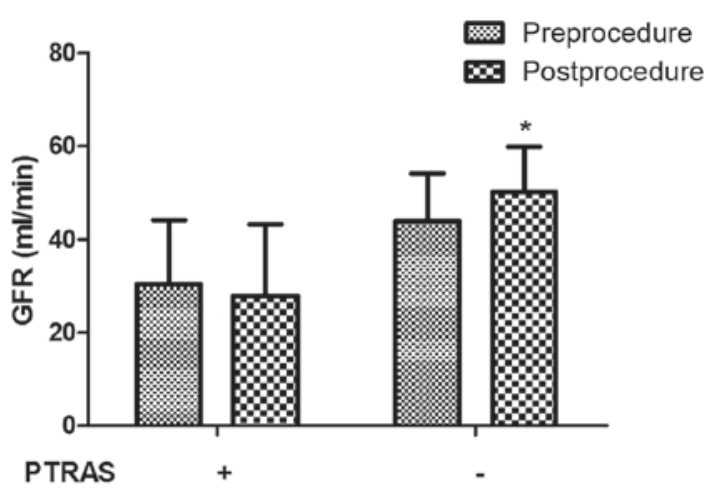

Figure 1. Comparison of GFR examined by ECT before and after PTRAS. There was no significant difference in the GFR of kidney on the side of implantation (PTRAS) before and after surgery $(\mathrm{P}>0.05)$, whereas the GFR of the kidney that did not receive PTRAS was significantly increased after surgery ( $\mathrm{P}=0.0014)$. GFR, glomerular filtration rate; PTRAS, percutaneous renal artery stent implantation.

renal function (21). Improvement in blood pressure control, promotion of cardiac function and renal blood supply are favorable factors for the prognosis of patients. The results of this study showed that PTRAS could effectively control the blood pressure, and block the progressive deterioration of renal function of patients, but it did not significantly improve the renal function of patients. Large-scale multi-center randomized controlled clinical trials have shown that the effect of renal artery intervention on the body is mainly manifested in blood pressure, and it has no significant effects on the protection of renal function and mortality rate $(15,17)$, which are similar to the results in this study. There may be several following reasons for this outcome brought by interventional therapy: i) in the absence of distal protection, interventional procedure may cause micro-thrombosis, cholesterol crystal embolism and contrast agent-induced renal damage, lead to impaired renal function and offset the benefit from interventional therapy; ii) renal artery stenosis is a long-term slow-onset lesion accompanied by long-term renal ischemia during the course, which can lead to irreversible changes, such as renal fibrosis and glomerular sclerosis; and iii) after interventional therapy, stenotic arterial blood flow is improved, and the renal perfusion pressure is increased; the high pressure and high perfusion can further accelerate the renal parenchymal damage. In this study, patients were followed up for 36 months, and the incidence rate of adverse cardiogenic or nephrogenic events was $24.3 \%$, 
Table V. OR value and 95\% CI of adverse events after PTRAS with different comorbidities.

\begin{tabular}{lccr}
\hline Comorbidity & Adverse event, $\mathrm{n}(\%)$ & OR $(95 \% \mathrm{CI})$ & P-value \\
\hline Sex & & & 0.644 \\
$\quad$ Male & $33(58.9)$ & $1.193(0.648-2.197)$ & \\
Female & $23(41.1)$ & & 0.005 \\
Age (years) & & & \\
$\geq 65$ & $41(73.2)$ & $2.610(1.347-5.060)$ & \\
$<65$ & $15(26.8)$ & & 0.039 \\
CCI score & & & $<0.001$ \\
1 & $3(5.4)$ & $4.136(1.084-15.780)$ & $<0.001$ \\
2 & $10(17.9)$ & $15.421(4.419-53.807)$ & 0.125 \\
3 & $29(51.8)$ & $28.424(7.001-115.325)$ & 0.023 \\
$\geq 4$ & $14(25)$ & $1.641(0.894-3.013)$ & $<0.001$ \\
Smoking history & $32(57.1)$ & $2.147(1.121-4.112)$ & 0.438 \\
Diabetes mellitus & $21(37.5)$ & $3.209(1.695-6.077)$ & $<0.001$ \\
Stroke & $26(46.4)$ & $0.758(0.414-1.388)$ & \\
Hyperlipidemia & $29(51.8)$ & $3.446(1.801-6.594)$ & \\
CHF & $25(44.6)$ & & \\
\hline
\end{tabular}

OR, odds ratio; CI, confidence interval; PTRAS, percutaneous renal artery stent implantation; CCI, Charlson comorbidity index; CHF, congestive heart failure.

which was lower than that in CORAL stent treatment group (35.1\%) (17). In addition, ARAS patients received renography in this study and results showed that GFR on the unaffected side, namely the kidney without receiving PTRAS, of patients with unilateral ARAS after PTRAS has no significant change, but GFR on the side receiving stent implantation was increased compared with that before PTRAS, which can be explained by the disappearance of a compensatory mechanism, but such reasons as progression of atherosclerosis or other accompanying diseases are not excluded.

Studies have shown that ARAS is often complicated with other atherosclerotic diseases, such as coronary heart disease, $\mathrm{CHF}$ and stroke, and these diseases jointly promote the progression of atherosclerosis $(22,23)$. In this study, multivariate regression analyses of adverse cardiogenic and nephrogenic events after PTRAS showed that the CCI score ( $\geq 2$ points), advanced age ( $\geq 65$ years old), diabetes mellitus, stroke and $\mathrm{CHF}$ are risk factors related to adverse cardiogenic and nephrogenic events in ARAS patients after PTRAS. Elderly patients with ARAS suffer from systemic function degradation, so its predictive value in adverse events after PTRAS is not unique. Among the various risk factors, CCI score $\geq 4$ points has the highest predictive value. In addition to correcting the renal artery stenosis, the optimal treatment of hypertension, diabetes mellitus, cerebrovascular diseases and other accompanying diseases can really improve the prognosis of patients; before the therapeutic regimen is developed for ARAS patients, the comorbidity of patients must be taken into full account, and the individualized therapeutic regimen should be developed (24-26).

A limitation for the current study is that this was a single-center retrospective analysis with limited number of patients enrolled, thus leading to a certain bias in the results.
In the future, the experimental design can be improved, the sample size can be expanded, and randomized controlled clinical trials can be performed, increasing the reliability of results.

In conclusion, PTRAS can effectively control the blood pressure, reduce the types of antihypertensive drugs used and prevent the deterioration of renal function of patients with ARAS, but it cannot improve the renal function of patients obviously. Age ( $\geq 65$ years old), CCI score ( $\geq 2$ points), diabetes mellitus, stroke and CHF are risk factors leading to adverse cardiogenic or nephrogenic events after PTRAS.

\section{Acknowledgements}

Not applicable.

\section{Funding}

No funding was received.

\section{Availability of data and materials}

All data generated or analyzed during this study are included in this published article.

\section{Authors' contributions}

YH contributed to the design of the study. YZ was responsible for the acquisition of the data, HW and YW for the data analysis, YY for the data interpretation, and YH for the preparation of the manuscript. CC and JL performed the patients' follow up. All authors read and approved the final manuscript. 


\section{Ethics approval and consent to participate}

This study was approved by the Ethics Committee of Taizhou Hospital of Traditional Chinese Medicine (Taizhou, China). Signed written informed consents were obtained from the patients and/or guardians.

\section{Patient consent for publication}

Not applicable.

\section{Competing interests}

The authors declare that they have no competing interests.

\section{References}

1. Safian RD: Atherosclerotic renal artery stenosis. Curr Treat Options Cardiovasc Med 5: 91-101, 2003.

2. Safian RD and Textor SC: Renal-artery stenosis. N Engl J Med 344: 431-442, 2001.

3. Tanemoto M, Saitoh H, Satoh F, Satoh H, Abe T and Ito S: Predictors of undiagnosed renal artery stenosis among Japanese patients with risk factors of atherosclerosis. Hypertens Res 28: 237-242, 2005

4. Zhang Y, Ge JB, Qian JY and Ye ZB: Prevalence and risk factors of atherosclerotic renal artery stenosis in 1,200 Chinese patients undergoing coronary angiography. Nephron Clin Pract 104 c185-c192, 2006.

5. Marcantoni C, Rastelli S, Zanoli L, Tripepi G, Di Salvo M, Monaco S, Sgroi C, Capodanno D, Tamburino C and Castellino P: Prevalence of renal artery stenosis in patients undergoing cardiac catheterization. Intern Emerg Med 8: 401-408, 2013.

6. Wang Y, Ho DS, Chen WH, Wang YQ, Lam WF, Shen ZJ, Lu CZ and Chui M: Prevalence and predictors of renal artery stenosis in Chinese patients with coronary artery disease. Intern Med J 33: 280-285, 2003.

7. Salehi N, Firouzi A, Gholoobi A, Shakerian F, Sanati HR, Ahmadabadi MN and Moradi M: Relationship between distribution of coronary artery lesions and renal artery stenosis in patients undergoing simultaneous coronary and renal angiography. Clin Med Insights Cardiol 5: 35-40, 2011.

8. Goldfarb DA: Prevalence of renovascular disease in the elderly: A population-based study. J Urol 170: 1053-1054, 2003.

9. Neves PD, Oliveira AA, Oliveira MC, Machado JR, Reis MA, Mendonça HM, Resende LA and Marques VP: Research of multiarterial atherosclerotic disease in hypertensive patients with renal artery stenosis. J Bras Nefrol 34: 243-250, 2012.

10. Textor SC: Atherosclerotic renovascular disease as a cause of end-stage renal disease: Cost considerations. Blood Purif 14: 305-314, 1996

11. Henry M, Benjelloun A, Henry I, Polydorou A and Hugel M: Renal angioplasty and stenting: Is it still indicated after ASTRAL and STAR studies? J Cardiovasc Surg (Torino) 51: 701-720, 2010.

12. Sarac TP: Influence and critique of the ASTRAL and CORAL trials. Semin Vasc Surg 24: 162-166, 2011.

13. Kanjwal K and Figueredo VM: Controversies in the management of the renal artery stenosis. Cardiol J 20: 11-16, 2013.

14. Bax L, Mali WP, Buskens E, Koomans HA, Beutler JJ, Braam B, Beek FJ, Rabelink TJ, Postma CT, Huysmans FT, et al; STAR Study Group: The benefit of STent placement and blood pressure and lipid-lowering for the prevention of progression of renal dysfunction caused by atherosclerotic ostial stenosis of the renal artery. The STAR-study: Rationale and study design. J Nephrol 16: 807-812, 2003.
15. Wheatley K, Ives N, Gray R, Kalra PA, Moss JG, Baigent C, Carr S, Chalmers N, Eadington D, Hamilton G, et al; ASTRAL Investigators: Revascularization versus medical therapy for renal-artery stenosis. N Engl J Med 361: 1953-1962, 2009.

16. Jaff MR, Bates M, Sullivan T, Popma J, Gao X, Zaugg M and Verta P; HERCULES Investigators: Significant reduction in systolic blood pressure following renal artery stenting in patients with uncontrolled hypertension: Results from the HERCULES trial. Catheter Cardiovasc Interv 80: 343-350, 2012.

17. Cooper CJ, Murphy TP, Cutlip DE, Jamerson K, Henrich W, Reid DM, Cohen DJ, Matsumoto AH, Steffes M, Jaff MR, et al; CORAL Investigators: Stenting and medical therapy for atherosclerotic renal-artery stenosis. N Engl J Med 370: 13-22, 2014.

18. Rundback JH, Sacks D, Kent KC, Cooper C, Jones D, Murphy T, Rosenfield K, White C, Bettmann M, Cortell S, et al; AHA Councils on Cardiovascular Radiology, High Blood Pressure Research, Kidney in Cardiovascular Disease, Cardio-Thoracic and Vascular Surgery, and Clinical Cardiology, and the Society of Interventional Radiology FDA Device Forum Committee: Guidelines for the reporting of renal artery revascularization in clinical trials. American Heart Association. Circulation 106: 1572-1585, 2002.

19. Campbell NR, Kaczorowski J, Lewanczuk RZ, Feldman R, Poirier L, Kwong MM, Lebel M, McAlister FA and Tobe SW; Canadian Hypertension Education Program: 2010 Canadian Hypertension Education Program (CHEP) recommendations: The scientific summary - an update of the 2010 theme and the science behind new CHEP recommendations. Can J Cardiol 26: 236-240, 2010

20. Yu TM, Sun CS, Lin CL, Wang CY, Chang PY, Chou CY, Chuang YW, Lee BJ and Kao CH: Risk factors associated with end-stage renal disease (ESRD) in patients with atherosclerotic renal artery stenosis: A nationwide population-based analysis. Medicine (Baltimore) 94: e912, 2015.

21. Uder M and Humke U: Endovascular therapy of renal artery stenosis: Where do we stand today? Cardiovasc Intervent Radiol 28: 139-147, 2005.

22. Chábová V, Schirger A, Stanson AW, McKusick MA and Textor SC: Outcomes of atherosclerotic renal artery stenosis managed without revascularization. Mayo Clin Proc 75: 437-444, 2000.

23. Wright JR, Shurrab AE, Cheung C, Waldek S, O'Donoghue DJ, Foley RN, Mamtora $\mathrm{H}$ and Kalra PA: A prospective study of the determinants of renal functional outcome and mortality in atherosclerotic renovascular disease. Am J Kidney Dis 39: 1153-1161, 2002.

24. Liu J, Huang Z, Gilbertson DT, Foley RN and Collins AJ: An improved comorbidity index for outcome analyses among dialysis patients. Kidney Int 77: 141-151, 2010.

25. Karanikas G, Becherer A, Wiesner K, Dudczak R and Kletter K: ACE inhibition is superior to angiotensin receptor blockade for renography in renal artery stenosis. Eur J Nucl Med Mol Imaging 29: 312-318, 2002.

26. Onuigbo MA: Evidence of the syndrome of rapid onset end-stage renal disease (SORO-ESRD) in the acute kidney injury (AKI) literature - preventable causes of AKI and SORO-ESRD - a call for re-engineering of nephrology practice paradigms. Ren Fail 35: 796-800, 2013.

This work is licensed under a Creative Commons Attribution-NonCommercial-NoDerivatives 4.0 International (CC BY-NC-ND 4.0) License. 